\title{
Estimating the demand for drop-off recycling sites: a random utility travel cost approach
}

\begin{abstract}
Drop-off recycling is one of the most widely adopted recycling programs in the United States. Despite its wide implementation, relatively little literature addresses the demand for drop-off recycling. This study examines the demand for drop-off recycling sites as a function of travel costs and various site characteristics using the random utility model (RUM). The findings of this study indicate that increased travel costs significantly reduce the frequency of visits to drop-off sites implying that the usage pattern of a site is influenced by its location relative to where people live. This study also demonstrates that site specific characteristics such as hours of operation, the number of recyclables accepted, acceptance of commingled recyclables, and acceptance of yard-waste affect the frequency of visits to drop-off sites.
\end{abstract}

Keyword: Drop-off centers; Drop-off recycling; Random utility model; Recycling; Travel cost 